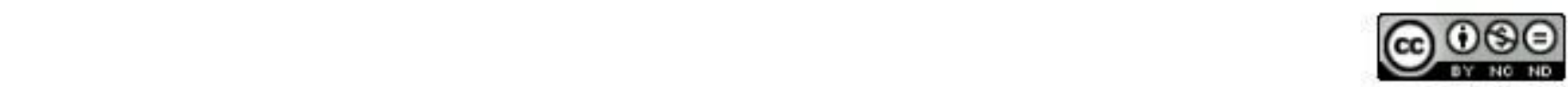

Trascender, Contabilidad y Gestión. Vol. 7, Núm. 19 (enero - abril del 2022).

Universidad de Sonora. Departamento de Contabilidad. México.

ISSN: 2448-6388. Reserva de Derechos 04-2015-04172070800-203.

\title{
Factores que influyen en la Gestión de la Innovación en empresas financiadas por el Gobierno Federal
}

\author{
Factors that influence Innovation Management in companies financed by Federal \\ Government
}

Leticia del Carmen Ríos Rodríguez $^{1}$; Eduardo Alejandro Carmona ${ }^{2}$; Leonel Ruvalcaba Arredondo ${ }^{3}$

Recibido: 25 de agosto de 2021.

Aceptado: 4 de octubre de 2021.

DOI: https://doi.org/10.36791/tcg.v7i19.133

JEL: O32. Gestión de la innovación tecnológica y de la I + D.

L25. Rendimiento de la empresa: tamaño, edad, beneficio y ventas.

M10. Administración de empresas (Generalidades).

\section{Resumen}

Entre las estrategias más eficientes para que una empresa logre una diferenciación favorable respecto a sus competidores está la innovación; al fomentarla, se promueve un óptimo desempeño social y económico. En esta investigación, se muestra el análisis de doce empresas participantes en el Programa de Estímulos a la Innovación (PEI) del Consejo Nacional de Ciencia y Tecnología (CONACYT), las cuales colaboraron con una Institución de Educación Superior (IES) en el Estado de Zacatecas, México, durante 2014-2017.

Esta entidad (Zacatecas) cuenta con poca incidencia

\footnotetext{
${ }^{1}$ Leticia del Carmen Ríos Rodríguez. Doctora en Ingeniería y Tecnología Aplicada. Docente - Investigador Unidad Académica Docencia Superior Universidad Autónoma de Zacatecas, Zacatecas, México. Correo: leticia.rios@uaz.edu.mx. ORCID: 0000-0002-1005-020X.

${ }^{2}$ Eduardo Alejandro Carmona. Doctor en Administración. Docente - Investigador Unidad Académica de Contabilidad y Administración, Universidad Autónoma de Zacatecas, Zacatecas, México. Correo: alexcar2001@uaz.edu.mx . ORCID: 0000-0002-3978-3052.

${ }^{3}$ Leonel Ruvalcaba Arredondo. Doctor en Administración. Docente - Investigador Unidad Académica Docencia Superior, Universidad Autónoma de Zacatecas, Zacatecas, México. Correo: l_ruvalcabaa@uaz.edu.mx. ORCID: 0000-0001-7031-8645.
}

Ríos Rodríguez, L. C., Carmona, E. A., y Ruvalcaba Arredondo, L.
Vol. 7, núm. 19 / enero - abril del 2022 DOI: https://doi.org/10.36791/tcg.v7i19.133

Pp. 37-67 
en proyectos de innovación, de ahí la pertinencia de este estudio cuyo objetivo consiste en determinar la varianza común del grupo de ítems estudiados que influyen en la gestión de la innovación de las empresas. Para conocer las variables de mayor impacto en la innovación, se diseñó un cuestionario estructurado con una escala tipo Likert. La información se analizó a través de análisis factorial (AF). A partir del análisis, se destaca que los factores: tecnología, competitividad y colaboración externa / vinculación, favorecen la gestión de la innovación dentro de las empresas. Se propone: una interfaz de comunicación entre las IES y la empresa, para facilitar la transferencia de tecnología y el reconocimiento mutuo; fomentar políticas gubernamentales que influyan en la mejora de competencias y capacidades de los recursos humanos y que promuevan la I\&D (Investigación y Desarrollo) en las empresas.

Palabras clave: gestión de la innovación, empresa, análisis factorial exploratorio (AFE).

\begin{abstract}
Among the most efficient strategies for a company to achieve a favorable differentiation from its competitors is innovation; by promoting it, optimal social and economic performance are promoted. This research shows the analysis of twelve SMEs (Small and medium enterprises) participating in the National Council of Science and Technology (CONACYT) Innovation Stimulus Program (PEI), which collaborated with a Higher Education Institution (IES) in the State of Zacatecas, Mexico, during 2014-2017. This entity (Zacatecas) has little impact on innovation projects. This entity (Zacatecas) has little impact on innovation projects, hence the relevance of this study whose objective is to determine the common variance of the group of items studied that influence the management of
\end{abstract}

innovation in companies. To know the variables with the greatest impact on innovation, a structured questionnaire with a Likert scale was designed. The information was analyzed through factor analysis (AF). From the analysis, it is highlighted that the factors: technology, competitiveness and external collaboration / linkage, favor the management of innovation within companies. Proposed: a communication interface between HEIs and the enterprise to facilitate technology transfer and mutual recognition; promote government policies

Keywords: innovation management, enterprise, Exploratory Factor Analysis.

\section{Introducción}

La innovación es un elemento esencial en la generación de ventajas competitivas de las empresas y también está comprobado su efecto positivo en la productividad de las mismas (Kato, 2019). En las últimas décadas, numerosos estudios (González y Mancillas, 2016; Ron y Sacoto 2017; Taborda, Nova y Bohórquez 2018) realzan la envergadura de las Micro, Pequeñas y Medianas empresas en la participación dentro del Producto Interno Bruto (PIB) del país, así como en la creación de empleos. En México, hay 4,169,677 empresas, mismas que general el 52\% del PIB y el 72\% del empleo (INEGI, 2018).

Dentro del Índice Global de Innovación 2019, México está en la posición número 56, lo que evidencia su lejana postura con respecto a los primeros lugares a nivel internacional (OMPI, 2019). Este dato constata que las empresas mexicanas tienen aún un área de oportunidad en cuanto a la mejora de los niveles de innovación. No obstante, dentro de los Estados de la República Mexicana, Zacatecas se encuentra en los últimos lugares en empresas que logran innovar, esto se demuestra al presentar el penúltimo lugar como Estado en número de 
proyectos del PEI del Consejo Nacional de Ciencia y Tecnología en el periodo de 2009 al 2018 (CONACYT, 2019). Asimismo, en la Tabla 1 se puede observar como en el Estado de Zacatecas el número de proyectos aprobados y vinculados con la UAZ son muy pocos en relación a los autorizados a nivel Nacional, tal como lo muestra la Tabla 1.

\section{Tabla 1}

Número de proyectos aprobados PEI de 2010 a 2018

\begin{tabular}{|c|c|c|}
\hline Año & Nivel Nacional & Zacatecas (vinculados con la UAZ) \\
\hline 2010 & 677 & 1 \\
\hline 2011 & 543 & 2 \\
\hline 2012 & 522 & 0 \\
\hline 2013 & 706 & 2 \\
\hline 2014 & 866 & 4 \\
\hline 2015 & 794 & 8 \\
\hline 2016 & 937 & 8 \\
\hline 2017 & 421 & 8 \\
\hline 2018 & 503 & 38 \\
\hline Total & 5969 & 5 \\
\hline
\end{tabular}

Fuente: Elaboración propia con información de CONACYT (2019).

De acuerdo con esta información, las causas de la falta de innovación de las empresas son evidentes. Uno de los elementos comunes observados en estas instancias es la escasa tecnología que poseen para su desempeño, situación que representa el problema por el que poseen una limitada competencia para ofrecer productos y servicios que se caractericen por tener un grado de innovación o distinción competitiva en su rama (Aranda y Solleiro, 2015). Asimismo, se ha identificado que las empresas carecen de estrategias y recursos para mantener un análisis sistemático y permanente dentro y fuera de las mismas, que les faculte valorar y decidir qué tipo de innovación adaptar, qué herramientas utilizar para lograrlo e identificar cuáles son los procesos organizacionales y de carácter administrativo, necesarios para llevar a cabo la transformación de los procesos y productos en las mismas (Mendoza y Valenzuela, 2013).

En este sentido, es ineludible adoptar una política de innovación, debido a que para lograr la competitividad se tiene que trabajar con ahínco y decisión, ya que esta no se obtiene por herencia, así como tampoco obedece de situaciones económicas (Simoeen, 2012; citado por Solleiro y Terán, 2012).
Ríos Rodríguez, L. C., Carmona, E. A., y Ruvalcaba Arredondo, L.
Vol. 7, núm. 19 / enero - abril del 2022 DOI: https://doi.org/10.36791/tcg.v7i19.133 
Esta construcción evolutiva del pensamiento administrativo despliega algunos traslapes entre teorías, mismos que posibilitan la visión multidisciplinaria y la integración de los conceptos para estudiar los factores que inciden en la innovación, por lo que se incursiona en la identificación de elementos de las diferentes teorías señaladas y su convergencia en un cuerpo teórico de mayor dimensión; también se explicitan los elementos metodológicos utilizados.

De acuerdo con los elementos expuestos, se analizan las situaciones que incitan a las empresas para: implementar procesos de innovación e identificar las particularidades de gestión de la innovación, sin soslayar que es importante examinar el proceso de las empresas beneficiadas por el CONACyT a través del PEI y la vinculación que de manera reciente han establecido las empresas beneficiadas con las Instituciones de Educación Superior (IES). El objeto de estudio son las 16 empresas apoyadas por el PEI en el periodo 20142017 vinculadas con la Universidad Autónoma de Zacatecas.

Estas condiciones nos llevan a plantearnos la siguiente pregunta de investigación: ¿Qué factores organizacionales son los que influyen primordialmente en la gestión de la innovación de las empresas apoyadas por el Programa de Estímulos a la Innovación del Consejo Nacional de Ciencia y Tecnología?

De este problema expresado nos orientamos al punto central de la investigación al expresar el objetivo siguiente:

Identificar los factores organizacionales que influyen primordialmente en la gestión de la innovación de las empresas apoyadas por el Programa de Estímulos a la Innovación del Consejo Nacional de Ciencia y Tecnología.
Esta meta que nos planteamos la resolveremos mediante la siguiente afirmación inicial expresada como hipótesis:

Las empresas apoyadas por el Programa de Estímulos a la Innovación del Consejo Nacional de Ciencia y Tecnología desarrollan factores organizacionales que influyen de manera determinante en la gestión de la innovación.

Este documento está compuesto de siete apartados: introducción, como se detalla líneas arriba, revisión de literatura donde se analiza la relación de la innovación con otras variables asociadas a teorías administrativas; posteriormente se describe el método utilizado aplicado al objeto de estudio (16 PyMES apoyadas por el PEI de CONACYT vinculadas por la UAZ) que comprendió desde el análisis de fiabilidad hasta el análisis factorial confirmatorio. Después se muestran los resultados y discusión de los mismos así como la identificación de hallazgos que se contrastan con las teorías referidas, al tiempo que se asume un posicionamiento desde los autores en mención para identificar elementos más complejos que pueden dar pie a teorías de mayor espectro exploratorio; finalmente se plantean algunas recomendaciones, así como los alcances, limitaciones y otras líneas de investigación.

\section{Revisión de literatura}

De la revisión de la literatura alusiva al tema de la innovación, se encontró que de ese concepto se derivan otros como tecnología, competitividad, colaboración externa, vinculación, cultura, propiedad intelectual, investigación y desarrollo, conocimiento y recursos humanos (Aranda y Solleiro, 2015; Aponte, 2016; Bukhkalo, Denysovets, Galagan y Dumenko, 2018; Camio, Rébori, y Romero, 2014; González, García, Caro y Romero, 2014; Haneda e Ito, 2018; Lecert y Omrani, 
2019; López, 2016; Maldonado y Martínez, 2012; Nieves y Quintana, 2016; Solleiro y Terán, 2012), conceptos que permiten comprender los procesos de innovación.

La palabra innovar deriva del latín innovare, que denota cambio de las cosas a través de la introducción o incorporación de novedades (Medina y Espinosa, 1994). Innovar significa "Mudar o alterar algo, introduciendo novedades” (Real Academia Española de la Lengua, 2019). El concepto entraña la “introducción de novedades”. El manual de Oslo define la innovación como "la concepción e implantación de cambios significativos en el producto, el proceso, el marketing o la organización de la empresa, con el propósito de mejorar los resultados” (Manual de Oslo, 2018). La innovación es también definida como la aptitud de la organización de modificar, extender o crear su área de recursos de manera intencional (Helfat, Finkelstein, Mitchell, Peteraf, Singh, Teece y Winter, 2017).

La institución que innova es la que desempeña nuevos objetos, brinda nuevos productos, transforma, progresa o incluye nuevos procedimientos de producción. (Escorsa y Valls, 2003). Pero en el caso, no se pretende como lo señalan Camio, Rébori y Romero (2014) clasificar la PyME como innovadora o no innovadora sino de distinguirla por su nivel de capacidad para innovar y desentrañar los elementos que la mantienen en esa condición.

Es evidente que el concepto contempla varios factores, entre ellos los insumos, la integración de tecnologías, uso de recursos y conocimientos e incluso las dinámicas que se gestan al interior de las instancias que hacen uso de la innovación; estos elementos pueden influir en mayor o menor medida en su gestión y son contemplados con frecuencia en los trabajos de investigación.

\section{Programa de Estímulos a la Innovación (CONACyT)}

El Programa de Estímulos a la Innovación (PEI) fue creado por el CONACyT a fin de fomentar innovaciones de productos, procesos o servicios, basadas en desarrollos tecnológicos, en las empresas mexicanas pertenecientes al Registro Nacional de Instituciones y Empresas Científicas y Tecnológicas (RENIECYT) que se dediquen a realizar prácticas de Investigación, Desarrollo Tecnológico e Innovación (IDTI) en México, ya sea de manera individual o vinculadas con Instituciones de Educación Superior (públicas o privadas) así como con Centros de Investigación Públicos Nacionales. Las empresas plantean un proyecto de desarrollo tecnológico, innovación tecnológica o investigación, mismo que es sometido a evaluación para verificar el cumplimiento de lineamientos y requisitos presentes en la convocatoria.

El programa se creó bajo tres modalidades:

1. INNOVAPYME (Innovación tecnológica para las micro, pequeñas y medianas empresas que pueden presentar solicitudes de forma individual o con vinculación con IES o Centros de Investigación (CI o ambos)

2. INNOVATEC (Innovación Tecnológica para las grandes empresas, cuyos proyectos pueden ser individuales o mediante la vinculación con IES, CI o ambos)

3. PROINNOVA (Proyectos en red orientados a la innovación, cuya propuesta debe vincularse con al menos dos CI, dos IES o uno de cada uno)
Ríos Rodríguez, L. C., Carmona, E. A., y Ruvalcaba Arredondo, L.
Vol. 7, núm. 19 / enero - abril del 2022 DOI: https://doi.org/10.36791/tcg.v7i19.133

Pp. $37-67$ 
Algunos de los objetivos del PEI son:

- El fomento del sector productivo a través del incremento por año de la inversión en IDTI. Aclarando que el apoyo es complementario, ya que la empresa tiene que poner otra parte del total de la inversión.

- Incentivar la vinculación de las empresas mediante la "educación-ciencia-tecnologíainnovación” y su enlace con el sector pertinente a través de la cadena de producción.

- La formación e incorporación de especializados recursos humanos dentro de las actividades pertenecientes al IDTI en las organizaciones.

- Crear productos, servicios y procesos nuevos en el mercado que posean un gran valor agregado y que promuevan la competitividad de la empresa.

- Generar propiedad intelectual en el país con acciones para el aseguramiento de su respectivo registro y protección ante las instituciones correspondientes.

- Incrementar el apoyo a las empresas nacionales de manera descentralizada.

Además de las cuestiones de forma, algunos de los criterios de evaluación y selección de proyectos bajo la modalidad PEI son los siguientes (no enlistados en orden de importancia):

- Vinculación con IES y CI

- Producción de Propiedad Intelectual

- Aumento en la productividad y creación de empleos de calidad y bien remunerados.

- Integración de empleados en las actividades de IDTI de la empresa que posean grados académicos desde Licenciatura hasta Doctorado.
- Vinculación de cadenas de producción en labores relacionadas a la IDTI.

- Priorizar estados o regiones cuyos sistemas de innovación requieran mayor vinculación.

- Dar preferencia a los sectores prioritarios de las entidades federativas.

(CONACYT, 2019)

De acuerdo a lo anterior, se pueden observar similitudes entre los factores que toma en cuenta el CONACYT para evaluar una solicitud o proyecto del PEI (tecnología, vinculación, propiedad intelectual, formación de recursos humanos, investigación y desarrollo) y las variables encontradas en la literatura que tienen relación con la gestión de la innovación, que para este estudio se describen enseguida:

\section{Innovación y Tecnología}

En la literatura revisada se manifiesta que a partir de la transferencia de conocimientos y tecnología, la interrelación de la organización y otros sujetos como clientes, proveedores, Centros de Investigación, e Instituciones de Educación Superior (IES) se produce una relación positiva e íntimamente ligada con la innovación (Becerra, Serna y Naranjo, 2013; Solleiro y Terán, 2012; Fernández, 2013; Aranda y Solleiro, 2015; Aranda, De la Fuente y Becerra, 2010; Jiménez, 2016; Mendoza y Valenzuela, 2013). Entre los agentes, las IES son esenciales para generar tecnología y conocimiento y, aunque no son consideradas como empresas una concepción literal, resultan importantes de acuerdo con un estudio realizado en Chile (Jiménez, 2016) en el que se manifiesta que estas se enfocan mayormente en la producción académica, protegida por derechos de autor y no se hace hincapié en la creación de tecnología, legalizada a través de patentes y/o aplicaciones industriales. Fernández (2013) resalta asimismo lo 
importante que es para la empresa contar con tecnología. Sin embargo, cabe mencionar que las empresas han adquirido técnicas de Gestión de Innovación Tecnológica de forma empírica (Aranda y Solleiro, 2015).

Una empresa se puede encontrar en determinado nivel de innovación y para que esta pueda realizar mejoras es imprescindible investigar, determinar y evaluar el nivel de innovación, todo esto como parte del desarrollo de la gestión de la innovación (Aranda, De la Fuente y Becerra, 2010; Camio, Rébori y Romero, 2014). No obstante ¿cómo reconocer el nivel de gestión de innovación tecnológica que posee una empresa? Aranda y su equipo proponen una herramienta creada en base a la concepción establecida por la Organización para la Cooperación y el Desarrollo Económicos (OCDE) y modelos de gestión tecnológica reconocidos, a fin de investigar y caracterizar el nivel de gestión que posee la organización, como los acontecimientos tecnológicos más importantes (Aranda, De la Fuente, y Becerra, 2010). Por otra parte, otros autores estudian las aptitudes que las PYMES han acumulado, así como su relación con respecto al nivel que tienen de gestión tecnológica (Mendoza y Valenzuela, 2013).

\section{Innovación y competitividad}

Dependiendo de la unidad que se analice, el indicador o la perspectiva que se aborde, el concepto de competitividad puede variar. Sin embargo, al referirse a la competitividad se hace referencia a varios conceptos de índole multidimensional, mismos que implican un aumento de la productividad, ya sea de organizaciones o naciones, la exportación de bienes y servicios y un uso de manera más eficaz de los recursos, lo anterior en pro del aumento de la calidad de vida de las personas (López, 2016).
En el entorno de esta investigación, se considera el término de competitividad en función del significado que le otorgue en mayor medida la empresa. La competitividad en la organización se basa en factores internos y externos; dentro de estos últimos el factor económico sí puede influir en ellos. Existen tres acciones que promueve la innovación: la generación de ventajas competitivas, el desarrollo de la economía y el crecimiento económico (Pérez y Góngora, 2016)

Cuando una empresa está inmersa en la gestión de la innovación y del conocimiento, logra poseer una ventaja competitiva, lo que produce satisfacción en sus clientes a través de los servicios o productos que ofrece (Aranda, De la Fuente y Becerra, 2010).

\section{Innovación y colaboración}

La colaboración es relevante para mejorar los resultados en algún proyecto; aunado al trabajo en equipo, esta combinación ha mostrado mayor rendimiento que el trabajo individual. La colaboración, utilizada a manera de plan empresarial posee un interés creciente entre los académicos e investigadores. Esto se advierte sobre todo en países en vías de desarrollo y se analiza su conexión con el proceso de la gestión de la innovación. Ahora bien, la repercusión que tiene la colaboración en la innovación en las empresas es un tema que falta por ser estudiado en las investigaciones recientes (López, Maldonado, Pinzón y García, 2015).

La gestión del conocimiento es muy beneficiosa para una PYME, debido a que se ha comprobado que deriva en efectos positivos en su desarrollo (Maldonado y Martínez, 2012). Es posible adquirir conocimiento dentro o fuera de la empresa, para este último objetivo se requiere, la colaboración con: Centros de Investigación, otras empresas y/o IES. Empresas de una
Ríos Rodríguez, L. C., Carmona, E. A., y Ruvalcaba Arredondo, L.
Vol. 7, núm. 19 / enero - abril del 2022 DOI: https://doi.org/10.36791/tcg.v7i19.133

Pp. $37-67$ 
misma localidad también pueden desarrollar y mantener una colaboración, impulsando así la creación de un clúster. Derivado de lo anterior, se puede tener como beneficio unir las actividades de innovación, tratados de cooperación en tecnología que permitan hacer más asequible un proyecto tecnológico, la transferencia del conocimiento, mediante de la creación de redes colectivas para la aplicación de desarrollo o fortalecimiento de procesos, productos o servicios (Fernández, 2013).

\section{Innovación y cultura organizacional}

La cultura organizacional se considera un componente esencial en la innovación de una empresa. La motivación de los empleados, la toma de decisiones de los directivos o la clase de jerarquía, contribuye o entorpece el proceso de gestión de la innovación. Hay empresas que dedican tiempo considerable de análisis y recursos a fin de impulsar la creatividad e innovación en los trabajadores y que ésta se vea plasmada en el servicio, producto o proceso de la organización. Se cree que la capacidad de innovar solo existe en grandes empresas, no obstante, las PyMES pueden desarrollar la gestión de la innovación, siempre que esta se considere como posibilidad de éxito.

En la cultura organizacional se alude a las particularidades y formas propias de la gestión, así como las normas que rigen a las instituciones, organismos o empresas, en el desarrollo de la toma de decisiones; al igual que las actividades organizacionales, se toman en cuenta elementos centrales como la comunicación, la toma de decisiones, la motivación y el control para lograr metas u objetivos propuestos (Camio, Rébori y Romero, 2014).

\section{Innovación y Propiedad Intelectual}

La propiedad intelectual es una actividad relacionada con las creaciones de la mente humana, estas pueden ser de diferentes campos del conocimiento, las tecnologías o las artes, que se traducen en obras literarias y artísticas, símbolos, nombres e imágenes utilizados en la mercadotecnia, diseño de software, entre otras; estas invenciones se rigen por lineamientos y principios gestados en la Organización Mundial de la Propiedad Intelectual (OMPI), que salvaguarda la protección legal a estas invenciones y para lograrlo establece un marco legal internacional para el trámite de patentes, derechos de autor, derechos de diseño, secretos comerciales, etc. Entre los propósitos se alude al impulso del crecimiento económico, por lo que resulta imprescindible la inversión para promover la innovación, sobre todo cuando esta atiende alguna problemática particular (comunidad) o general (sociedad). Entre los problemas más sentidos que enfrenta la innovación, es que la mayoría de estas, sobre todo en las empresas, no legalizan su propiedad intelectual, aunado a que un número importante de solicitudes relacionadas con la autoría de la propiedad intelectual no finalizan el trámite y otras tantas que logran legalizar su autoría, no son aprovechadas en el mercado. Conocedores de este problema y de acuerdo con sus investigaciones, Monzón (2015) y Aranda y Solleiro (2015) demuestran que en México existen vacíos en la protección del patrimonio intelectual de las PyMES.

\section{Innovación, Investigación y Desarrollo}

Las empresas que logran invertir en actividades de Investigación y Desarrollo (I\&D) tienen una relación positiva con la innovación (Becerra, Serna y Naranjo, 2013; Monzón, 2015; Palacios y Saavedra, 2016). El problema es que muy pocas empresas invierten en I\&D, en específico las PyMES, las cuales muestran capacidades limitadas, en parte porque sus necesidades más inmediatas son de otra naturaleza (infraestructura tecnológica de orden fiscal) y para desarrollar I\&D es importante la inversión, condición que se refleja en sus 
escasos procesos de innovación.

\section{Innovación y Conocimiento}

Generar y transferir conocimiento forman parte de las capacidades que promueven la innovación. Esto se puede generar dentro de la empresa, entre trabajadores, directivos, etc., como fuera de la misma con proveedores, clientes, otras empresas, IES y gobierno. Para fomentar las actividades de innovación, desarrollo, transferencia de tecnología y conocimiento se promueven las redes empresariales, entre las que figuran los clusters (Becerra, Serna y Naranjo, 2013). La adquisición, socialización del conocimiento y la acumulación de competencias tecnológicas, juegan un rol importante y tienen influencia positiva en la mejora de procesos de producción (Büttenbender, 2019).

\section{Innovación y Recursos Humanos}

Los recursos humanos es un activo importante dentro de la estructura de una empresa, por lo que es indispensable descubrir la forma adecuada de compensarlos, motivarlos, seleccionarlos, capacitarlos, reclutarlos y remunerarlos según las actividades que realizan. Un factor que resalta es la motivación ya que es clave para que las empresas implementen estrategias. Los directivos y trabajadores determinan su influencia en los procesos de innovación según el nivel de compromiso y motivación de éstos (Solleiro y Castañón, 2015). Idealmente estas variables se hacen presentes en los procesos de innovación, de ahí la importancia de ubicarlas en las PyMES para definir el modelo organizacional.

\section{Metodología}

El objeto de estudio fue la totalidad de empresas apoyadas por el PEI (en uno o varios proyectos) en el periodo 2014-2017 (Tabla 2), que son un total de 16. Tal como se señaló en la Tabla 1 líneas arriba, en este estudio se toma en cuenta todas las empresas beneficiadas, que si bien es cierto son pocas, aunado a ello se tuvo el inconveniente que a pesar de las insistencias, 4 de ellas no respondieron, lo que evidencia la problemática del acceso a la información por parte del investigador. No obstante, cabe señalar que las empresas analizadas simbolizan el prototipo de espacios en los que la innovación representa una meta, motivo por el cual participan en dicho programa y se vinculan a nivel local.

En base a la literatura revisada se realizó una operacionalización de variables, identificando las más representativas en relación a su nivel de impacto en la gestión de la innovación. Posteriormente se realizó un cuestionario, en el que se plasman desde 1 hasta 4 preguntas por cada variable analizada, que en total derivó en un instrumento de 68 ítems, contando con una evaluación de escala tipo Likert con cinco posiciones. El instrumento se aplicó a gerentes o directivos de las empresas mencionadas. El proceso de levantamiento de la información llevó tres semanas, obteniendo 12 instrumentos contestados. Aunado a este proceso, se entrevistaron a aquellos directivos de empresas cuya participación en el PEI ha sido en más de una ocasión (muestreo de juicio), dando un total de tres directivos entrevistados; la intención de este complemento metodológico fue conocer más a fondo su proceso en la gestión de la innovación y lograr explicaciones más profundas sobre su estructura organizacional.
Ríos Rodríguez, L. C., Carmona, E. A., y Ruvalcaba Arredondo, L.
Vol. 7, núm. 19 / enero - abril del 2022 DOI: https://doi.org/10.36791/tcg.v7i19.133

Pp. 37-67 
Tabla 2

Proyectos beneficiados en el Programa de Estímulos a la Innovación del 2014 al 2017 vinculados con la Universidad Autónoma de Zacatecas

\begin{tabular}{|c|c|c|c|c|}
\hline $\begin{array}{c}\text { No. } \\
\text { Empresa }\end{array}$ & Giro & Proyecto & Sede & $\begin{array}{c}\text { Convocatoria } \\
\text { participante }\end{array}$ \\
\hline 1 & $\begin{array}{l}\text { Desarrollo de } \\
\text { productos para el } \\
\text { sector agroindustrial } \\
\end{array}$ & $\begin{array}{l}\text { Desarrollo de agrotecnología para } \\
\text { producción hortícola intensiva. }\end{array}$ & $\begin{array}{l}\text { Ciudad de } \\
\text { México }\end{array}$ & 2015 \\
\hline $\begin{array}{c}\text { No. } \\
\text { Empresa }\end{array}$ & Giro & Proyecto & Sede & $\begin{array}{c}\text { Convocatoria } \\
\text { participante }\end{array}$ \\
\hline 2 & $\begin{array}{l}\text { Mantenimiento } \\
\text { Mecánico }\end{array}$ & $\begin{array}{l}\text { Sistema de Administración y Control de } \\
\text { Mantenimiento predictivo, preventivo y } \\
\text { correctivo para la Industria Minera } \\
\text { (Maquinaria de Molienda y Flotación). }\end{array}$ & Zacatecas & 2017 \\
\hline 3 & $\begin{array}{l}\text { Acuacultura y } \\
\text { Biotecnología }\end{array}$ & $\begin{array}{l}\text { Equipo biotecnológico automatizado para } \\
\text { preservar tejidos animales con fines de } \\
\text { investigación para exportación. }\end{array}$ & Querétaro & 2015 \\
\hline 4 & $\begin{array}{l}\text { Acuacultura y } \\
\text { Biotecnología }\end{array}$ & $\begin{array}{l}\text { Desarrollo del Método de Inclusión para } \\
\text { preservación de tejidos para el mercado de } \\
\text { exportación hacia los EUA. }\end{array}$ & Querétaro & 2016 \\
\hline \multirow{2}{*}{5} & \multirow{2}{*}{$\begin{array}{l}\text { Venta productos } \\
\text { para minería }\end{array}$} & $\begin{array}{l}\text { Sistema modular de seguridad y } \\
\text { monitoreo con infraestructura de bajo } \\
\text { costo para la industria minera. }\end{array}$ & \multirow{2}{*}{ Zacatecas } & 2014 \\
\hline & & $\begin{array}{l}\text { Desarrollo de sistema de monitoreo con } \\
\text { módulos de optimización de procesos y } \\
\text { recursos para la minería. }\end{array}$ & & 2015 \\
\hline \multirow{2}{*}{6} & \multirow{2}{*}{$\begin{array}{l}\text { Desarrollo de } \\
\text { tecnologías de la } \\
\text { información }\end{array}$} & $\begin{array}{l}\text { Plataforma aérea no tripulada de medio } \\
\text { alcance para monitoreo ambiental, } \\
\text { agrícola, e inspección. }\end{array}$ & \multirow{2}{*}{ Zacatecas } & 2015 \\
\hline & & $\begin{array}{l}\text { Sistema tecnológico integral E-Commerce } \\
\text { WEB y móvil basado en realidad } \\
\text { aumentada. }\end{array}$ & & 2017 \\
\hline 7 & $\begin{array}{l}\text { Fabricación de pisos } \\
\text { y azulejos }\end{array}$ & $\begin{array}{l}\text { Innovación y Desarrollo de Tecnología } \\
\text { Nacional para la fabricación industrial de } \\
\text { cerámicos de nueva generación. }\end{array}$ & Zacatecas & 2017 \\
\hline
\end{tabular}


Tabla 2

Continuación...

\begin{tabular}{|c|c|c|c|c|}
\hline $\begin{array}{c}\text { No. } \\
\text { Empresa }\end{array}$ & Giro & Proyecto & Sede & $\begin{array}{c}\text { Convocatoria } \\
\text { participante }\end{array}$ \\
\hline \multirow[t]{2}{*}{8} & \multirow[t]{2}{*}{ Sistema de riego } & $\begin{array}{l}\text { Prototipo de un sistema automático para } \\
\text { potenciar la gestión hidráulica y de } \\
\text { nutrición en sistemas agrícolas, enfocado } \\
\text { al uso eficiente del agua y cuidado del } \\
\text { suelo. }\end{array}$ & \multirow[t]{2}{*}{ Zacatecas } & 2014 \\
\hline & & $\begin{array}{l}\text { Diseño y construcción de un prototipo de } \\
\text { cámara de enfriamiento solar por } \\
\text { absorción continuo. }\end{array}$ & & 2016 \\
\hline 9 & Venta de huevo & $\begin{array}{l}\text { Producción intensiva e industrialización } \\
\text { de subproductos de sábila usando } \\
\text { innovación tecnológica en la zona noreste } \\
\text { de Guanajuato. }\end{array}$ & Guanajuato & 2015 \\
\hline 10 & $\begin{array}{l}\text { Desarrollo } \\
\text { tecnológico }\end{array}$ & $\begin{array}{l}\text { Diseño, desarrollo y puesta en marcha de } \\
\text { un prototipo sistema de radiografía digital } \\
\text { multimodal para servicios odontológicos. }\end{array}$ & Durango & 2017 \\
\hline 11 & $\begin{array}{l}\text { Desarrollo y } \\
\text { comercio de equipo } \\
\text { instrumental, } \\
\text { médico y de } \\
\text { laboratorio } \\
\end{array}$ & $\begin{array}{l}\text { Desarrollo y validación de un nuevo } \\
\text { nutracéutico para el tratamiento del } \\
\text { Síndrome Metabólico. Parte II }\end{array}$ & $\begin{array}{l}\text { Ciudad de } \\
\text { México }\end{array}$ & 2017 \\
\hline 12 & $\begin{array}{l}\text { Desarrollo de } \\
\text { productos para el } \\
\text { sector agroindustrial }\end{array}$ & $\begin{array}{l}\text { Planta Piloto para la Producción de } \\
\text { Alimentos Bajo Condiciones Controladas } \\
\text { para Ciudades Inteligentes. }\end{array}$ & Querétaro & 2016 \\
\hline \multirow[t]{2}{*}{13} & \multirow[t]{2}{*}{$\begin{array}{l}\text { Fabricación de } \\
\text { carrocerías y } \\
\text { remolques }\end{array}$} & $\begin{array}{l}\text { Innovación tecnológica en semi- } \\
\text { remolques tipo volteo para carga pesada } \\
\text { basados en nuevos materiales, procesos de } \\
\text { manufactura avanzada y diseño } \\
\text { electrónico moderno. }\end{array}$ & \multirow[t]{2}{*}{ Zacatecas } & 2015 \\
\hline & & $\begin{array}{l}\text { Innovación y desarrollo tecnológico en } \\
\text { semi-remolques tipo volteo para carga } \\
\text { pesada. }\end{array}$ & & 2016 \\
\hline
\end{tabular}

Ríos Rodríguez, L. C., Carmona, E. A., y Ruvalcaba Arredondo, L.
Vol. 7, núm. 19 / enero - abril del 2022

DOI: https://doi.org/10.36791/tcg.v7i19.133

Pp. $37-67$ 
Tabla 2

Continuación...

\begin{tabular}{|c|c|c|c|c|}
\hline $\begin{array}{c}\text { No. } \\
\text { Empresa }\end{array}$ & Giro & Proyecto & Sede & $\begin{array}{c}\text { Convocatoria } \\
\text { participante }\end{array}$ \\
\hline 13 & $\begin{array}{l}\text { Fabricación de } \\
\text { carrocerías y } \\
\text { remolques }\end{array}$ & $\begin{array}{l}\text { Innovación y desarrollo tecnológico en } \\
\text { sistemas integrales para Semi-Remolques } \\
\text { automatizados tipo volteo para carga } \\
\text { pesada para el Sector Minero. }\end{array}$ & Zacatecas & 2017 \\
\hline 14 & $\begin{array}{l}\text { Instalaciones } \\
\text { Eléctricas }\end{array}$ & $\begin{array}{l}\text { Diseño e implementación de un } \\
\text { dispositivo electrónico para medir la } \\
\text { fertilidad en mujeres mediante } \\
\text { procesamiento de imágenes de muestras } \\
\text { de saliva. }\end{array}$ & Querétaro & 2016 \\
\hline \multirow{3}{*}{15} & \multirow{3}{*}{$\begin{array}{l}\text { Desarrollo } \\
\text { telecomunicaciones }\end{array}$} & $\begin{array}{l}\text { Sistema de Flujo operativo mediante } \\
\text { identificación por radiofrecuencia para la } \\
\text { industria minera. }\end{array}$ & \multirow{3}{*}{ Zacatecas } & 2015 \\
\hline & & $\begin{array}{l}\text { Sistema experto de seguridad y } \\
\text { administración de proyectos para la } \\
\text { industria minera. }\end{array}$ & & 2016 \\
\hline & & $\begin{array}{l}\text { Segunda etapa del sistema experto de } \\
\text { seguridad para la industria minera basado } \\
\text { en identificación por radiofrecuencia } \\
\text { empleando espectro disperso. }\end{array}$ & & 2017 \\
\hline \multirow{3}{*}{16} & \multirow{3}{*}{$\begin{array}{l}\text { Desarrollo de } \\
\text { tecnologías de la } \\
\text { información }\end{array}$} & $\begin{array}{l}\text { Diseño, desarrollo y puesta en marcha de } \\
\text { una plataforma WEB y móvil interactiva } \\
\text { con arquitectura de hardware basada en } \\
\text { RA, RV, QR. }\end{array}$ & Durango & 2017 \\
\hline & & $\begin{array}{l}\text { Detección oportuna de enfermedades en } \\
\text { ganado bovino y toma de decisiones en } \\
\text { tiempo real para el incremento de la } \\
\text { productividad y competitividad del Sector } \\
\text { Agropecuario en México. }\end{array}$ & \multirow[t]{2}{*}{ Sinaloa } & 2015 \\
\hline & & $\begin{array}{l}\text { Diseño de sensores aplicados a la } \\
\text { detección y pronóstico de enfermedades } \\
\text { del ganado bovino para la industria } \\
\text { agropecuaria en México. }\end{array}$ & & 2016 \\
\hline
\end{tabular}

Fuente: Elaboración propia en base a información obtenida. 
Para analizar la información obtenida, se utiliza el Análisis Factorial Exploratorio con el objetivo de determinar la varianza común del grupo de ítems estudiados (Lloret, Ferreres, Baeza y Tomás, 2014). El modelo que se empleó para estimar la saturación de las variables fue el de Factorización de Ejes Principales (método de estimación iterativo). Igualmente, los factores se rotaron con el método Varimax con el objetivo de maximizar la varianza de éstos, además de minimizar la cantidad de ítems debido a que se seleccionan los que tienen una saturación alta en los factores puesto que se optimiza la solución de cada uno de éstos. El objetivo del método seleccionado es poder analizar y explicar de manera más concisa los resultados, es decir, se buscó una agrupación de las variables para comprobar su representatividad (varianza común) en relación a la gestión de la innovación, a partir de la generación de combinaciones lineales (Factores) de variables muy correlacionadas (ítems correlacionados). El número de factores es considerablemente menor que el número de variables originales, el cual da la opción para que los factores sean ortogonales, es decir, que sean independientes uno del otro (De la Fuente, 2019; Tabachnick y Fidell, 2007). La Tabla 3 indica las agrupaciones de los ítems para la elaboración del AFE.

\section{Tabla 3}

Dimensiones para el Análisis Factorial

\begin{tabular}{|c|c|c|c|c|}
\hline Factor & Ítems & Subvariables & Variables & Dimensión \\
\hline \multirow{3}{*}{1} & \multirow{3}{*}{1 al 8} & Comunicación & \multirow{3}{*}{ Administración } & \multirow{10}{*}{$\begin{array}{c}\text { Modelo } \\
\text { Organizacional }\end{array}$} \\
\hline & & Motivación & & \\
\hline & & Reclutamiento & & \\
\hline \multirow{2}{*}{2} & \multirow{2}{*}{$10-12 ; 24-25$} & Gestión Organizacional & Gestión & \\
\hline & & Software & Eficiencia & \\
\hline \multirow{5}{*}{3} & \multirow{5}{*}{$13-22$} & Trab. Colab. Interno & \multirow{2}{*}{ Equipos de Trabajo } & \\
\hline & & Políticas de Apoyo & & \\
\hline & & Productividad & \multirow{2}{*}{ Calidad } & \\
\hline & & Confiabilidad & & \\
\hline & & Sistematización & Eficiencia & \\
\hline
\end{tabular}

Ríos Rodríguez, L. C., Carmona, E. A., y Ruvalcaba Arredondo, L.
Vol. 7, núm. 19 / enero - abril del 2022 DOI: https://doi.org/10.36791/tcg.v7i19.133 


\section{Tabla 3}

Continuación...

\begin{tabular}{|c|c|c|c|c|}
\hline Factor & Ítems & Subvariables & Variables & Dimensión \\
\hline \multirow{3}{*}{4} & \multirow{3}{*}{$26-36$} & Tecnología & \multirow{7}{*}{ Innovación } & \multirow{8}{*}{$\begin{array}{l}\text { Modelo Gestión de } \\
\text { la Innovación }\end{array}$} \\
\hline & & Competitividad & & \\
\hline & & Colaboración & & \\
\hline \multirow{2}{*}{5} & \multirow{2}{*}{$37-43$} & Cultura & & \\
\hline & & Propiedad Intelectual & & \\
\hline \multirow{2}{*}{6} & \multirow{2}{*}{$44-54$} & Investigación y Desarrollo & & \\
\hline & & Conocimiento & & \\
\hline \multirow{4}{*}{7} & \multirow{4}{*}{$55-59 ; 62-64$} & $\mathrm{RH}$ & Innovación & \\
\hline & & Ventas & IE Económicos & \multirow{6}{*}{$\begin{array}{l}\text { Indicadores } \\
\text { Empresariales }\end{array}$} \\
\hline & & Clima Laboral & IE Capital & \\
\hline & & Rotación de Personal & Humano & \\
\hline \multirow{3}{*}{8} & \multirow{3}{*}{$60-61 ; 65-67$} & Utilidades & IE Económicos & \\
\hline & & Ausentismo & $\begin{array}{ll}\text { IE } & \text { Capital } \\
\text { Humano } & \\
\end{array}$ & \\
\hline & & Resp. Social empresa & IE Sociales & \\
\hline
\end{tabular}

Fuente: Elaboración propia en base a bibliografía consultada.

\section{Resultados}

La Tabla 4 expone las 8 dimensiones (grupos) que se agruparon y analizaron conforme a los ítems de cada variable, así como también la varianza de cada factor, asimismo se eliminaron los reactivos que presentaron cargas factoriales menores a 0.30 . Lo anterior expuesto indica que la explicación de la varianza tiene un valor mayor a 50\%, el único que está por debajo del porcentaje es el Grupo III que es de 49.7\%; lo anterior significa que el modelo tiene una buena explicación de las varianzas. 


\section{Tabla 4}

Proporción de la varianza explicada de los factores por grupo analizado

\begin{tabular}{|c|c|c|c|}
\hline No. Factor & $\begin{array}{l}\text { Varianza explicada / } \\
\text { factor }\end{array}$ & $\begin{array}{l}\text { Varianza } \\
\text { explicada / } \\
\text { grupo }\end{array}$ & No. Grupo \\
\hline F1 & 60.49 & \multirow{2}{*}{82.6} & \multirow{2}{*}{$\begin{array}{l}\text { Grupo I (Comunicación, motivación y } \\
\text { reclutamiento) }\end{array}$} \\
\hline F2 & 22.15 & & \\
\hline F3 & 35.97 & \multirow{2}{*}{64.4} & \multirow{2}{*}{ Grupo II (Gestión organizacional y software) } \\
\hline F4 & 28.42 & & \\
\hline F5 & 33.44 & \multirow{3}{*}{77.1} & \multirow{3}{*}{$\begin{array}{c}\text { Grupo III (Trabajo colaborativo interno, políticas } \\
\text { de apoyo, productividad, confiabilidad y } \\
\text { sistematización) }\end{array}$} \\
\hline F6 & 27.44 & & \\
\hline F7 & 16.26 & & \\
\hline F8 & 39.38 & \multirow{4}{*}{96.8} & \multirow{4}{*}{$\begin{array}{l}\text { Grupo IV (Tecnología, competitividad y } \\
\text { colaboración externa / vinculación) }\end{array}$} \\
\hline F9 & 24.82 & & \\
\hline F10 & 16.72 & & \\
\hline F11 & 15.90 & & \\
\hline No. Factor & $\begin{array}{l}\text { Varianza explicada / } \\
\text { factor }\end{array}$ & $\begin{array}{l}\text { Varianza } \\
\text { explicada / } \\
\text { grupo }\end{array}$ & No. Grupo \\
\hline F12 & 39.38 & \multirow{3}{*}{78.9} & \multirow{3}{*}{ Grupo V (Cultura y propiedad intelectual) } \\
\hline F13 & 20.54 & & \\
\hline F14 & 18.94 & & \\
\hline F15 & 23.68 & \multirow{4}{*}{71.7} & \multirow{4}{*}{$\begin{array}{c}\text { Grupo VI (Conocimiento e Investigación y } \\
\text { Desarrollo) }\end{array}$} \\
\hline F16 & 23.56 & & \\
\hline F17 & 14.56 & & \\
\hline F18 & 9.89 & & \\
\hline F19 & 51.63 & \multirow{2}{*}{71.0} & \multirow{2}{*}{$\begin{array}{l}\text { Grupo VII (Recursos Humanos, indicadores } \\
\text { empresariales económicos y administrativos) }\end{array}$} \\
\hline F20 & 19.33 & & \\
\hline F21 & 40.75 & \multirow{2}{*}{72.6} & \multirow{2}{*}{$\begin{array}{l}\text { Grupo VIII (Indicadores empresariales } \\
\text { económicos, administrativos y sociales) }\end{array}$} \\
\hline F22 & 31.89 & & \\
\hline
\end{tabular}

Fuente: Elaboración propia en base al análisis obtenido en SPSS.

Ríos Rodríguez, L. C., Carmona, E. A., y Ruvalcaba Arredondo, L. 
Finalmente, se ordenan los grupos por su grado de varianza explicada, para determinar qué variables poseen mayor influencia en el modelo y se realiza el análisis de fiabilidad a través del Alpha de Cronbach (Revelle y Zinbarg, 2009; Taber, 2018).

\section{Comunalidades}

En la tabla 5 se presentan las comunalidades de cada dimensión. El método de factorización de ejes principales permite generar extracciones de comunalidades que sustituyen a las anteriores con el fin de satisfacer el criterio de convergencia para la extracción a partir de los cambios en las comunalidades de una iteración a otra. Estas están agrupadas conforme al análisis por ítems explicado en la tabla 3. Se puede observar que, en general, la proporción de la varianza explicada de cada una de las variables es alta, pero se resalta las que tienen valor de 0.8 en adelante.

Tabla 5

Comunalidades

\begin{tabular}{|l|l|l|}
\hline Dimensión 1. Comunicación, motivación y reclutamiento & Inicial & Extracción \\
\hline 1. (Comunicación) Se comunican los procesos de innovación en tiempos adecuados & ,850 & $\mathbf{, 8 7 2}$ \\
\hline $\begin{array}{l}\text { 2. (Comunicación) Existe una buena recepción de los mensajes acerca de innovación } \\
\text { por parte de la dirección }\end{array}$ &, 896 & $\mathbf{, 8 1 2}$ \\
\hline $\begin{array}{l}\text { 3. (Comunicación) La empresa toma en cuenta las opiniones de los empleados acerca } \\
\text { de innovación }\end{array}$ &, 911 &, 737 \\
\hline $\begin{array}{l}\text { 4. (Comunicación) A los miembros de la organización se les facilita manifestar las } \\
\text { ideas de innovación. }\end{array}$ &, 814 &, 643 \\
\hline 5. (Motivación) Los empleados están motivados para innovar &, 912 &, 663 \\
\hline \begin{tabular}{l} 
6. (Motivación) Se les recompensa a los empleados por sus ideas de innovación \\
\hline $\begin{array}{l}\text { 7. (Motivación) La empresa tiene diferentes maneras de motivar a los empleados para } \\
\text { innovar }\end{array}$
\end{tabular} &, 484 &, 235 \\
\hline 8. (Reclutamiento) La empresa recluta a empleados que propician la innovación &, 796 &, 938 \\
\hline Dimensión 2. Gestión organizacional y software & Inicial & Extracción \\
\hline 10. (Gest Org) La empresa cuenta con acceso a diferentes fuentes de financiamiento &, 427 &, 532 \\
\hline 11. (Gest Org) La empresa cuenta con un plan de crecimiento &, 312 & ,325 \\
\hline $\begin{array}{l}\text { 12. (Gest Org) La innovación ha impactado de manera positiva en el crecimiento de la } \\
\text { empresa }\end{array}$ &, 389 & ,482 \\
\hline
\end{tabular}




\section{Tabla 5}

Continuación...

\begin{tabular}{|c|c|c|}
\hline Dimensión 2. Gestión organizacional y software & Inicial & Extracción \\
\hline $\begin{array}{l}\text { 24. (Software) Se cuenta con el software adecuado para realizar todos los procesos de } \\
\text { producción }\end{array}$ & ,797 & ,916 \\
\hline 25. (Software) Se ha desarrollado software específico para los procesos de innovación & ,768 & ,965 \\
\hline \multicolumn{3}{|l|}{ Método de extracción: Factorización de Ejes principales. } \\
\hline $\begin{array}{l}\text { Dimensión 3. Trabajo colaborativo interno, políticas de apoyo, productividad, } \\
\text { confiabilidad y sistematización }\end{array}$ & Inicial & Extracción \\
\hline $\begin{array}{l}\text { 13. (Trab Equipo) Los trabajadores prefieren trabajar en equipos que de manera } \\
\text { individual. }\end{array}$ & ,977 & ,456 \\
\hline 14. (Trab Equipo) Los trabajadores de la empresa son proactivos & ,967 & ,779 \\
\hline $\begin{array}{l}\text { 15. (Polit Apoyo) Las políticas públicas para fomentar la innovación que propone el } \\
\text { gobierno han tenido un impacto en la empresa. }\end{array}$ & ,989 & ,999 \\
\hline $\begin{array}{l}\text { 16. (Polit Apoyo) La empresa aprovecha los apoyos externos para vincularse con otras } \\
\text { empresas }\end{array}$ & ,992 & ,555 \\
\hline 17. (Polit Apoyo) La empresa invierte en otros proyectos de innovación además del PEI & ,981 &, 579 \\
\hline $\begin{array}{l}\text { 18. (Polit Apoyo) Estoy satisfecho con el apoyo otorgado por CONACyT a través del } \\
\text { PEI }\end{array}$ & ,990 & ,386 \\
\hline 19. (Productividad) La empresa es más productiva porque innova & ,991 & ,963 \\
\hline 20. (Productividad) El tiempo invertido en innovación se ve reflejado en productividad & ,949 & ,661 \\
\hline $\begin{array}{l}\text { 21. (Confiabilidad) Los productos o procesos de innovación han generado confiabilidad } \\
\text { en nuestros clientes }\end{array}$ & ,987 & ,805 \\
\hline $\begin{array}{l}\text { 22. (Sistematización) Todos los procesos de la organización están sistematizados y } \\
\text { documentados bajo manuales }\end{array}$ & ,984 & ,672 \\
\hline \multicolumn{3}{|l|}{ Dimensión 4. Tecnología, competitividad y colaboración externa / vinculación } \\
\hline $\begin{array}{l}\text { 15. (Polit Apoyo) Las políticas públicas para fomentar la innovación que propone el } \\
\text { gobierno han tenido un impacto en la empresa. }\end{array}$ & ,601 & ,585 \\
\hline $\begin{array}{l}\text { 16. (Polit Apoyo) La empresa aprovecha los apoyos externos para vincularse con otras } \\
\text { empresas }\end{array}$ & ,678 & ,494 \\
\hline 17. (Polit Apoyo) La empresa invierte en otros proyectos de innovación además del PEI & ,633 & ,682 \\
\hline
\end{tabular}

Ríos Rodríguez, L. C., Carmona, E. A., y Ruvalcaba Arredondo, L. 
Tabla 5

Continuación...

\begin{tabular}{|l|l|l|}
\hline Dimensión 4. Tecnología, competitividad y colaboración externa / vinculación & & \\
\hline $\begin{array}{l}\text { 18. (Polit Apoyo) Estoy satisfecho con el apoyo otorgado por CONACyT a través del } \\
\text { PEI }\end{array}$ &, 595 &, 394 \\
\hline $\begin{array}{l}\text { 21. (Confiabilidad) Los productos o procesos de innovación han generado confiabilidad } \\
\text { en nuestros clientes }\end{array}$ &, 500 &, 567 \\
\hline 20. (Productividad) El tiempo invertido en innovación se ve reflejado en productividad &, 573 &, 782 \\
\hline Método de extracción: factorización de eje principal. & & \\
\hline Dimensión 5. Cultura y propiedad intelectual & Inicial & Extracción \\
\hline 37. (Cultura) La cultura de innovación en sus trabajadores es buena &, 361 &, 404 \\
\hline $\begin{array}{l}\text { 38. (Cultura) Existe diferencia de cultura de innovación en sus empleados dependiendo } \\
\text { del nivel jerárquico }\end{array}$ &, 693 & $\mathbf{, 8 7 1}$ \\
\hline $\begin{array}{l}\text { 39. (Cultura) Las estrategias de mejora de productos/procesos/servicios han sido bien } \\
\text { aceptadas por los empleados }\end{array}$ &, 840 & $\mathbf{, 9 0 9}$ \\
\hline 40. (Cultura) Dentro de la organización se innova regularmente &, 711 &, 739 \\
\hline $\begin{array}{l}\text { 41. (Cultura) Los empleados suelen manifestar sus ideas de innovación de } \\
\text { productos/procesos/servicios con confianza }\end{array}$ &, 737 & $\mathbf{, 9 1 0}$ \\
\hline 42. (Prop Int) La empresa patenta regularmente &, 868 & $\mathbf{, 9 1 4}$ \\
\hline 43. (Prop Int) Las patentes de la empresa han generado regalías &, 726 &, 772 \\
\hline Dimensión 6. Conocimiento e Investigación y Desarrollo & Inicial & Extracción \\
\hline $\begin{array}{l}\text { 44. (Inv y Des) La empresa invierte en Investigación y Desarrollo anualmente } \\
\text { 45. (Inv y Des) La empresa invierte tiempo, dinero y esfuerzo en el diseño constante de } \\
\text { sus productos/procesos/servicios. }\end{array}$ &, 696 &, 410 \\
\hline $\begin{array}{l}\text { 46. (Inv y Des) Los diseños de sus productos/procesos/servicios toman en cuenta las } \\
\text { necesidades del cliente }\end{array}$ &, 851 & $\mathbf{, 9 2 6}$ \\
\hline $\begin{array}{l}\text { 47. (Inv y Des) La empresa acude regularmente a organizaciones que cuentan con } \\
\text { centros de investigación. }\end{array}$ &, 757 & $\mathbf{, 8 0 9}$ \\
\hline
\end{tabular}




\section{Tabla 5}

Continuación...

\begin{tabular}{|c|c|c|}
\hline Dimensión 5. Cultura y propiedad intelectual & Inicial & Extracción \\
\hline 37. (Cultura) La cultura de innovación en sus trabajadores es buena & ,361 & ,404 \\
\hline $\begin{array}{l}\text { 38. (Cultura) Existe diferencia de cultura de innovación en sus empleados dependiendo } \\
\text { del nivel jerárquico }\end{array}$ & 693 & ,871 \\
\hline $\begin{array}{l}\text { 39. (Cultura) Las estrategias de mejora de productos/procesos/servicios han sido bien } \\
\text { aceptadas por los empleados }\end{array}$ & ,840 & ,909 \\
\hline 40. (Cultura) Dentro de la organización se innova regularmente & ,711 & ,739 \\
\hline $\begin{array}{l}\text { 41. (Cultura) Los empleados suelen manifestar sus ideas de innovación de } \\
\text { productos/procesos/servicios con confianza }\end{array}$ & ,737 & ,910 \\
\hline 42. (Prop Int) La empresa patenta regularmente & ,868 &, 914 \\
\hline 43. (Prop Int) Las patentes de la empresa han generado regalías & ,726 & ,772 \\
\hline Dimensión 6. Conocimiento e Investigación y Desarrollo & Inicial & Extracción \\
\hline 44. (Inv y Des) La empresa invierte en Investigación y Desarrollo anualmente & ,696 & ,410 \\
\hline $\begin{array}{l}\text { 45. (Inv y Des) La empresa invierte tiempo, dinero y esfuerzo en el diseño constante de } \\
\text { sus productos/procesos/servicios. }\end{array}$ & ,909 & ,929 \\
\hline $\begin{array}{l}\text { 46. (Inv y Des) Los diseños de sus productos/procesos/servicios toman en cuenta las } \\
\text { necesidades del cliente }\end{array}$ & ,851 & ,826 \\
\hline $\begin{array}{l}\text { 47. (Inv y Des) La empresa acude regularmente a organizaciones que cuentan con } \\
\text { centros de investigación. }\end{array}$ & ,757 & ,809 \\
\hline $\begin{array}{l}\text { 48. (Conocimiento) Todos los productos/procesos que realiza la empresa proceden de } \\
\text { una base científica más que empírica }\end{array}$ & ,740 & ,370 \\
\hline $\begin{array}{l}\text { 49. (Conocimiento) La empresa se preocupa por adquirir o generar nuevos } \\
\text { conocimientos }\end{array}$ & ,738 & ,677 \\
\hline 50. (Conocimiento) La empresa adquiere nuevos conocimientos de manera periódica & ,772 & 887 \\
\hline $\begin{array}{l}\text { 51. (Conocimiento) Los empleados están capacitados para hacer uso de los materiales, } \\
\text { equipo y procesos que se emplean }\end{array}$ & ,894 & ,858 \\
\hline 53. (Conocimiento) Los empleados son creativos & ,270 & ,268 \\
\hline 54. (Conocimiento) La empresa fomenta que los empleados tengan nuevas ideas & ,690 & ,371 \\
\hline
\end{tabular}

Ríos Rodríguez, L. C., Carmona, E. A., y Ruvalcaba Arredondo, L. 


\section{Tabla 5}

Continuación...

\begin{tabular}{|l|l|l|}
\hline $\begin{array}{l}\text { Dimensión 7. Recursos Humanos, indicadores empresariales económicos y } \\
\text { administrativos }\end{array}$ & Inicial & Extracción \\
\hline $\begin{array}{l}\text { 55. (RH) La empresa ha realizado acciones para evitar que los buenos empleados se } \\
\text { retiren de ella }\end{array}$ &, 901 & $\mathbf{, 8 9 5}$ \\
\hline 56. (RH) La empresa tiene trabajadores que innovan con regularidad &, 629 &, 730 \\
\hline $\begin{array}{l}\text { 57. (RH) La empresa cuenta con las mejores condiciones físicas para que los } \\
\text { empleados puedan innovar }\end{array}$ &, 821 &, 392 \\
\hline $\begin{array}{l}\text { 58. (RH) La empresa promueve acciones para que los empleados se sientan satisfechos } \\
\text { con sus empleos }\end{array}$ &, 751 &, 619 \\
\hline $\begin{array}{l}\text { 59. (Economía empresa) Hay un incremento en las ventas de la empresa a partir de su } \\
\text { participación en el Programa de Estímulos a la Innovación }\end{array}$ &, 889 &, 662 \\
\hline $\begin{array}{l}\text { 62. (Economía empresa) Con la participación en el PEI, la relación entre pasivos y } \\
\text { capital ha mejorado }\end{array}$ &, 921 &, 674 \\
\hline 63. (Admon empresa) El clima laboral de su empresa es favorable &, 843 &, 419 \\
\hline 64. (Admon empresa) La rotación de personal de su empresa es baja &, 898 &, 537 \\
\hline Dimensión 8. Gestión organizacional y software & Inicial & Extracción \\
\hline $\begin{array}{l}\text { 60. (Economía empresa) La empresa muestra utilidades derivado de sus procesos de } \\
\text { innovación aplicados }\end{array}$ &, 420 &, 555 \\
\hline $\begin{array}{l}\text { 61. (Economía empresa) En el mercado hay algún producto innovador de su empresa } \\
\text { derivado de su participación en el PEI }\end{array}$ &, 664 &, $\mathbf{8 6 4}$ \\
\hline 65. (Admon empresa) No existe ausentismo en la empresa &, 637 &, 693 \\
\hline 66. (Admon empresa) Siempre existe trabajo colaborativo en su empresa &, 343 &, 406 \\
\hline $\begin{array}{l}\text { 67. (Social empresa) La empresa realiza algún programa de responsabilidad social } \\
\text { como parte de sus actividades }\end{array}$ &, 385 &, 554 \\
\hline Método de extracción: Factorización de Ejes principales. & , & \\
\hline
\end{tabular}

Fuente: Elaboración propia. 


\section{Análisis de Fiabilidad}

Para el caso se utilizó la opción Alfa de Cronbach, pues permite medir una cantidad de ítems mediana con un número pequeño de encuestas. La expresión de cálculo es:

$$
\alpha=\frac{k}{k-1}\left[1-\frac{\sum_{i=1}^{68} 5 i^{2}}{5 t^{2}}\right]
$$

Por lo que resulta:

$\alpha=\frac{68}{67}\left[\begin{array}{cc}1 & 4484848 \\ 509.455\end{array}\right]=0.92$

De acuerdo a lo expuesto por George y Mallery, se contó con un instrumento de alta confiabilidad.

\section{Análisis Factorial}

El método de rotación ortogonal Varimax se empleó para extraer los principales factores, lo cual se realizó con el software SPSS. La relevancia de cada factor se expresa a través del porcentaje de varianza explicada. Después de que se determinaron la cargas factoriales de las 68 variables, así como los porcentajes de varianza asociados a cada uno de los factores, se dispone del porcentaje total que explica la combinación de factores, esta solución (número de factores) está determinada por los factores con eigenvalores (valor propio) mayores que 1.

La carga factorial que se consideró para que sea parte de una dimensión fue que su valor sea mayor a 0.45 . Las variables 13, 52 y 53 tienen valores de 0.372, -0.004 y 0.302 respectivamente. Las varianzas mayores a $50 \%$ son los resultados que destacan. Lo anterior es un indicativo de que el modelo tiene una buena explicación a través del análisis factorial, por lo que se estudian los factores que poseen un mayor efecto, mismos que se observan en la Tabla 6.

\section{Tabla 6}

Proporción de la varianza de los factores por grupo analizado

\begin{tabular}{|c|l|c|}
\hline No. & \multicolumn{1}{|c|}{ Factor } & $\begin{array}{c}\text { Varianza explicada por } \\
\text { factor (\%) }\end{array}$ \\
\hline 1 & Tecnología, competitividad y colaboración externa / vinculación & 96.8 \\
\hline 2 & Comunicación, motivación y reclutamiento & 82.6 \\
\hline 3 & Cultura y propiedad intelectual & 78.9 \\
\hline 4 & $\begin{array}{l}\text { Trabajo colaborativo interno, políticas de apoyo, productividad, } \\
\text { confiabilidad y sistematización }\end{array}$ & 77.1 \\
\hline 5 & Indicadores empresariales económicos, administrativos y sociales & 72.6 \\
\hline 6 & Conocimiento e Investigación y Desarrollo & 71.7 \\
\hline 7 & $\begin{array}{l}\text { Recursos Humanos, indicadores empresariales económicos y } \\
\text { administrativos }\end{array}$ & 71.0 \\
\hline 8 & Gestión organizacional y software & 64.4 \\
\hline
\end{tabular}

Fuente: Elaboración propia.

Ríos Rodríguez, L. C., Carmona, E. A., y Ruvalcaba Arredondo, L.
Vol. 7, núm. 19 / enero - abril del 2022 DOI: https://doi.org/10.36791/tcg.v7i19.133 
El análisis factorial expuso que las variables: tecnología, competitividad y colaboración externa / vinculación son las que tiene un mayor efecto sobre la innovación. De tal forma que las empresas que se analizaron innovan debido a que usan tecnología empleando la transferencia de la misma y el conocimiento para adquirirla (Soto, Popa y Martínez, 2018), lo cual se liga a la coexistencia de vinculación y colaboración con los proveedores, clientes, centros de investigación y otras empresas. Asimismo, las empresas participantes en esta investigación son consideradas competitivas puesto que buscan tener ventajas comparativas y de esta forma obtener preferencia de los clientes (Pérez y Góngora 2016).

\section{La comunicación, motivación y reclutamiento} pertenecen al segundo factor que más influencia tienen en la innovación. La combinación de estos elementos hace que los individuos estén motivados en el proyecto, lo que les permite mejorar la comunicación y alcanzar las metas que puede ser un proyecto nuevo, innovador. Esto coincide con Camio, Rébori y Romero (2014) que analizan que las empresas con mayor grado de innovación poseen elementos comunes que favorecen la generación sostenida de la gestión de la innovación, como la motivación, comunicación y una promoción sostenida de la cultura organizacional.

En el tercer factor se observa una varianza que se puede explicar en un $78.9 \%$, la cual incluye las variables de cultura y propiedad intelectual, siendo la cuarta y quinta variables con mayor influencia en la innovación. Dentro de este factor las acciones que tienen más influencia en fomentar la innovación al interior de la empresa son el promover y analizar las propuestas que permiten mejorar el proceso, producto o servicio por parte de los trabajadores, las acciones constantes de innovación, la creación de regalías y patentes, que en total generan un $39.4 \%$ del total de la varianza explicada. Lo que es seguido por el nivel cultural relativo a la innovación de los empleados de acuerdo a la jerarquía en la organización con 20.5\% y la manifestación las ideas por parte de los trabajadores en $18.9 \%$.

El cuarto factor comprende el trabajo de colaboración al interior de la organización, las políticas que apoyan a la empresa, la productividad, el proceso de confiabilidad y la sistematización con varianza explicada de $77.1 \%$. En este grupo se observan 9 preguntas (ítems) agrupados en sólo tres factores. Si se colocan de acuerdo a la relevancia están la confiabilidad y la productividad en un $33.4 \%$ del total explicado de $77.1 \%$; las políticas que apoyan a las empresas lo hacen con $27.4 \%$, así como el nivel de proactividad de los integrantes de la organización, después aparecen la vinculación con otras organizaciones y la sistematización que influyen con un 16.3\% de la explicación de la varianza. Estos grupos forman parte de lo que se ha definido como Modelo organizacional e indican primordialmente que la innovación de las empresas que se han analizado promueve la productividad así como el fomento de la confianza de los clientes en la empresa. Al respecto $\mathrm{Fu}$, Mohnen y Zanello (2017) confirman que la innovación impacta positivamente la productividad laboral de las empresas.

El quinto factor comprende los indicadores empresariales relativos a la economía; así como los indicadores administrativos y los sociales. En conjunto presentan un valor de la varianza que se puede explicar de $72.6 \%$. Este componente muestra un incremento en las ganancias de las empresas que se analizaron a través de su participación en el PEI. Este resultado coincide con el estudio que realizan $\mathrm{Li}$ y su grupo de colaboradores (Li, Zhenga, Cao, Chen, Ren y Huang, 2017). Cabe destacar también que las iniciativas de capacitación, los centros de especialización y el 
desarrollo empresarial promueven la innovación (Aswegen y Retief, 2020).

Las variables investigación y desarrollo, así como conocimiento conforman el sexto factor que aportan una varianza explicada de $71.7 \%$ por cada factor; que presenta una diferencia pequeña de $0.9 \%$ con el factor anterior. Dentro de la literatura revisada Becerra, Serna y Naranjo (2013), Monzón (2015) y Palacios y Saavedra (2016) destacan una relación positiva entre las variables investigación y desarrollo y conocimiento y su influencia en la capacidad de innovar de una empresa.

El séptimo factor con una varianza total explicada de $71 \%$ (sólo $0.7 \%$ por debajo del anterior) contiene la variable en la que se encuentran recursos humanos y también algunos indicadores empresariales, así como económicos. En la variable donde se cuestiona acerca de los recursos humanos podemos identificar lo más representativo del grupo, pues cuenta con 52\% de los $71 \%$ de la varianza total explicada. Por otro lado, las variables que tienen que ver con los indicadores empresariales, administrativos y sociales aportan un porcentaje de $10 \%$. Es de destacar que los recursos humanos definitivamente son parte importante del proceso innovativo. Khan, Hussain, Shahbaz, Yang y Jiao, (2020) coinciden en que el recurso humano afecta positivamente el desarrollo financiero y la innovación. El 50\% de las organizaciones encuestadas mencionaron que tuvieron un incremento en sus ventas después de su participación en el PEI (sin especificar los porcentajes que generaron); y otro 50\% de ellas mencionaron que sí hubo crecimiento en este rubro, pero menores que las anteriores. También se observa la importancia de permitir innovar a los trabajadores de una manera regular y se les debe de fomentar estas condiciones. Es de destacar que se importante reducir la relación entre pasivo y capital, así como la rotación de personal. No se debe dejar de lado la importancia de capacitar a los trabajadores, fomentar las competencias en términos de conocimiento mediante sus destrezas y habilidades que se adquieren en ciencia y tecnología para desarrollar con el tiempo (Cimoli, 2000).

Lo que se ha encontrado como factor número ocho, toma por un lado la gestión organizacional y el software, con una varianza de $64 \%$. Cuando se toma en cuenta el factor que influye en el software, se presenta con una varianza de $35.9 \%$, por encima de aquel que incluye la gestión organizacional con un $28.4 \%$. Así se observa que, aunque no es determinante, es muy importante poseer un software apropiado que facilite los procesos que se dan en el interior de la organización. Al analizar a las 12 empresas estudiadas, tan sólo dos de ellas, expresaron que cuentan con el software adecuado para realizar los procesos de producción adecuados; también expresan que han desarrollado programas específicos para los procesos de innovación. Tres empresas están en un nivel alto en lo que a desarrollo y adquisición de software se refiere, mientras que las otras siete expresan carencia en estas circunstancias. Por otra parte la investigación arrojó que la gestión organizacional está ligada a la cultura organizacional, también a la investigación y desarrollo y por último a la innovación y al desarrollo tecnológico. Al realizar la visita a las empresas con más de una participación en el PEI, los directivos afirmaron que la motivación del empresario aunado a la colaboración de la organización en actividades de investigación y desarrollo constituyen factores determinantes en el nivel de innovación de la empresa, esta afirmación concuerda con Martínez y Romero (2017).

\section{Conclusiones y recomendaciones}

En la revisión de la literatura relacionada con este
Ríos Rodríguez, L. C., Carmona, E. A., y Ruvalcaba Arredondo, L.
Vol. 7, núm. 19 / enero - abril del 2022 DOI: https://doi.org/10.36791/tcg.v7i19.133

Pp. $37-67$ 
tema, hay investigaciones (Camio, Rébori y Romero, 2014; González, García, Caro y Romero, 2014; González y Mancilla, 2016; Hsu, 2016; Jiménez, 2016; López, 2016; Monzón, 2015; Solleiro y Castañón, 2015; Solleiro y Terán, 2012; Pérez y Góngora, 2016) que enfatizan en la relación existente de la innovación con variables que impactan de manera positiva en las organizaciones. Entre las citadas variables destacamos que las que tienen mayor impacto con condiciones acordes en la influencia en el proceso de innovación en las empresas (Figura 1).

\section{Figura 1}

Bases del desarrollo de la innovación

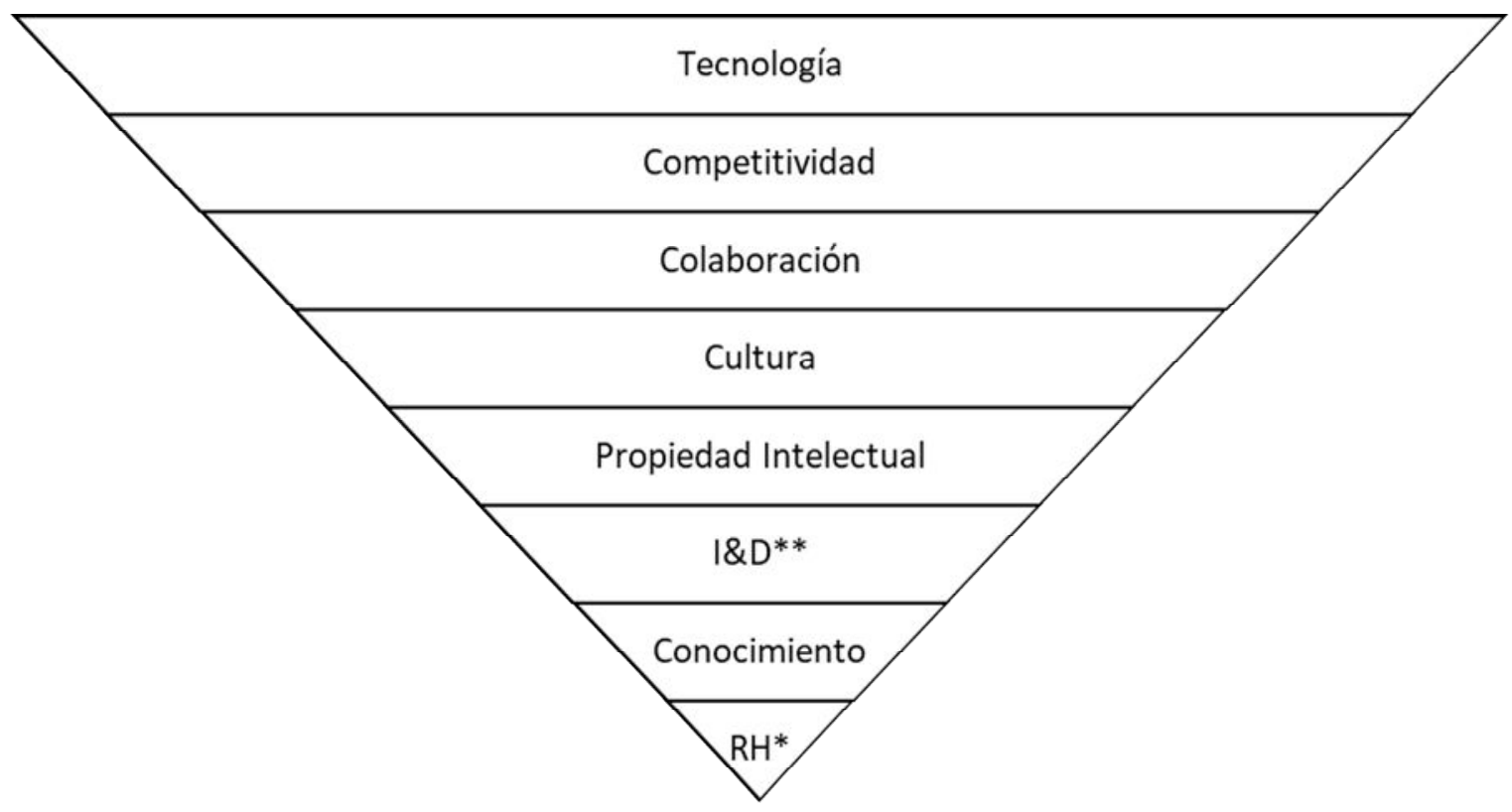

*R.H. Recurso Humano

**I\&D: Investigación y Desarrollo

Fuente: Elaboración propia con base en la información recabada. 
Eso concuerda en mayor medida con los resultados obtenidos en esta investigación referidos en la tabla 6, con los cuales es posible expresar la convergencia de elementos que son determinantes en la gestión de la innovación, mismos que, con otros estudios posteriores, podrían resultar como componentes para una teoría resultante que facilite la visión de la multidisciplina relacionada con la innovación y contar con un modelo base para operar con las empresas en los procesos de gestión de la innovación, que considere las siguientes variables: i) Modelo organizacional, ii) Modelo que permite gestionar la innovación y iii) Modelo que toma en cuenta los indicadores empresariales.

En consecuencia, resulta impostergable la promoción de la innovación en las empresas, en particular en el estado de Zacatecas. Esto se puede observar en la Figura 2, que explica cómo es que las condiciones para que existan comunicación, motivación y un adecuado reclutamiento dependen de los recursos humanos, el software, el conocimiento generado y la I\&D a través del trabajo colaborativo. A estas condiciones se le debe de agregar la cultura organizacional y la generación de la propiedad intelectual que son detonantes para la existencia de tecnología, competitividad y colaboración con el exterior. El resultado de estas variables conjuntadas es una organización innovadora que impacta de una manera favorable en sus indicadores económicos, sociales y administrativos.
Ríos Rodríguez, L. C., Carmona, E. A., y Ruvalcaba Arredondo, L.
Vol. 7, núm. 19 / enero - abril del 2022 DOI: https://doi.org/10.36791/tcg.v7i19.133 


\section{Figura 2}

Modelo de Gestión Organizacional para la Innovación en las Pymes

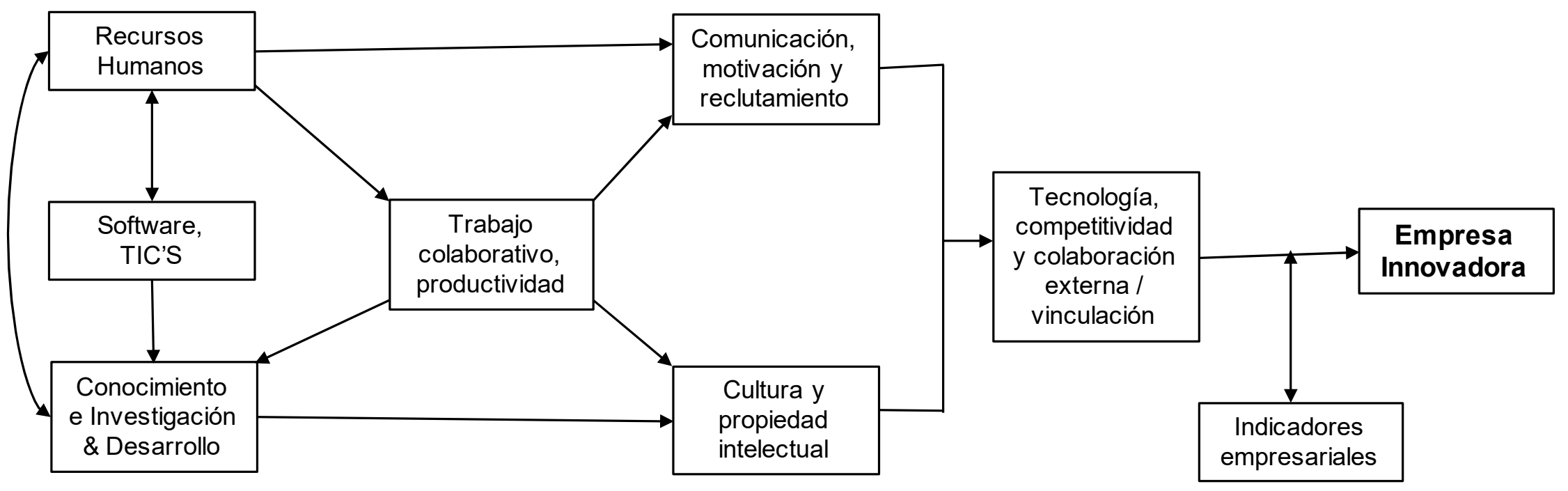

Fuente: Elaboración propia con base en literatura revisada. 
Con el objetivo de que las organizaciones cuenten con mayor I\&D se propone una interfaz que permita comunicar a quien está generando el conocimiento y el usuario, es decir, un espacio administrativo para poder conectar el proceso de la transferencia tecnológica y el conocimiento de la industria en la entidad.

A fin de contar con personal en las empresas que sea competente en ciencia y tecnología, resulta importante fomentar políticas por parte de los organismos gubernamentales que tengan impacto en el desarrollo de competencias, habilidades y destrezas del personal involucrado; tener Instituciones Educativas de calidad, que promuevan la I\&D y que cuenten con sistemas de producción, es decir, personal que tenga experiencia en procesos productivos $\mathrm{y}$ en base a esto pueda ser contratado para que adquieran y lleven los procesos en esta dirección; competencias que fueron adquiridas en el sistema de educación; resultados que concuerdan con Cimoli (2000). Se recomienda que los empleados busquen estrategias para que realicen su trabajo motivados, remunerados y con oportunidades verdaderas de crecer al interior de la organización. Se debe contar con un programa enfocado a mejorar organizacionalmente el clima de la empresa, así como la cultura de la misma para que vayan enfocados a la innovación.

A partir de los resultados obtenidos en este trabajo, se puede observar que para el tiempo y el conjunto de organizaciones estudiadas, el PEI permitió que las organizaciones inicien con las técnicas de innovación, por lo que se justifica la necesidad de enfatizar en la promoción de la innovación en las empresas en las políticas gubernamentales.

Bajo estas condiciones podemos decir que contamos con evidencia para no rechazar la hipótesis expresada al inicio del trabajo que menciona: "Las empresas apoyadas por el Programa de Estímulos a la Innovación del Consejo Nacional de Ciencia y Tecnología desarrollan factores organizacionales que influyen de manera determinante en la gestión de la innovación” pues se concluyó que existen al menos siete factores que permiten de una manera adecuada desarrollar estrategias que los conduzcan a procesos de innovación al contar con un programa gubernamental similar.

Por lo tanto, se puede concluir, en base a la hipótesis planteada, que las empresas analizadas muestran elementos idóneos que aluden a comunicación adecuada y propicias técnicas de motivación, tomando en cuenta efectivos procesos de reclutamiento, esta condición las ubica como empresas que muestran elementos que permiten mejorar en la gestión de la innovación.

\section{Alcances, limitaciones y futuras líneas de investigación}

No en todas las variables se explica el $100 \%$ de la varianza. Para poder explicar el mayor valor de la varianza tendríamos que tomar en cuenta las siguientes opciones: volver a plantear el instrumento expresando preguntas más claras, contar con al menos 4 ítems por variable y facilitar información a los respondientes para que les ayude a expresar las respuestas y no inducir la ponderación hacia las mismas, asó como analizar a más organizaciones beneficiadas con el PEI vinculadas con otros Centros de Investigación u otras IES. Algo a considerar es que los coeficientes de correlación tienden a ser menos confiables cuando la muestra es pequeña y, aunque lo ideal es contar con más empresas en estudiadas, este análisis factorial puede ser útil ya que es significativo y se pueden identificar un conjunto interpretable de factores, independientemente del nivel de medición de la entrada datos (Pearson y Mundform, 2010).
Ríos Rodríguez, L. C., Carmona, E. A., y Ruvalcaba Arredondo, L.
Vol. 7, núm. 19 / enero - abril del 2022 DOI: https://doi.org/10.36791/tcg.v7i19.133

Pp. 37-67 
Para futuras líneas de investigación se pretende realizar un Análisis de Clúster, técnica multivariante cuyo principal propósito es agrupar nuestro objeto de estudio formando conglomerados de las mismas con un alto grado de homogeneidad interna y heterogeneidad externa a fin de proponer acciones de política pública con alto grado de precisión de acuerdo a los perfiles identificados, lo cual podría asegurar mejores resultados. Una vez que se realice la réplica de los datos, se podrá obtener una base de datos que facilite obtener parámetros que permitan contrastarse con el modelo estudiado; lo que podría sugerir que si se cuenta con un número suficiente de datos se podría realizar un análisis bajo la técnica de Ecuaciones Estructurales.

\section{Referencias}

Aponte, G. (2016). Gestión de la Innovación

Tecnológica mediante el análisis de la información de patentes. Revista Negotium Científica Electrónica de Ciencias Gerenciales, 33(11), 42-68.

Aranda, H., De la Fuente, M., y Becerra, M. (2010). Propuesta metodológica para evaluar la Gestión de la Innovación Tecnológica (GIT) en pequeñas y medianas empresas (PYMES), Revista Mexicana de Agronegocios, 26(1), 226-238.

Aranda, H., y Solleiro, J. (2015). Gestión de la innovación tecnológica en Pymes Agroindustriales Chihuahuenses, Revista Mexicana de Agronegocios, 23(1), 681-694.

Aswegen, M., y Retief, F. (2020). The role of innovation and knowledge networks as a policy mechanism towards more resilient peripheral regions. Land Use Policy, 90, 1-12. https://doi.org/10.1016/j.landusepol.2019.104259
Bukhkalo, S., Denysovets, I., Galagan, N., y Dumenko, H. (2018). Intellectual Property as Complex Innovation Projects Component. International Journal of Engineering \& Technology, 7(4.8), 596-602. http://dx.doi.org/10.14419/ijet.v7i4.8.27313

Büttenbender, L., Zamberlan, A., y Sparemberge, L. (2009). Management of innovation, performance and added value: a case study in the agrobusiness chain, Revista Visión de Futuro, 12(2).

Becerra, F., Serna, H., y Naranjo, J. (2013). Redes empresariales locales, investigación y desarrollo e innovación en la empresa. Clúster de herramientas de Caldas, Colombia, Estudios Gerenciales, 29, 247-257. http://dx.doi.org/10.1016/j.estger.2013.05.013

Camio, M., Rébori, A., y Romero, M. (2014). Gestión de la Innovación. Estudio de casos en empresas de software y servicios electrónicos de la zona de influencia de la UNICEN, Argentina. Revista de Administración e Innovación, 11(2), 30-50. https://doi.org/10.5773/rai.v11i2.962

Cimoli, M. (2000). Developing Innovation Systems: México in Global Context. England. Continuum.

Consejo Nacional de Ciencia y Tecnología (CONACYT). (2019). Gobierno de la República Mexicana. https://www.conacyt.gob.mx/index.php/fondos-yapoyos/programa-de-estimulos-a-la-innovacion

De la Fuente, S. (2019). Análisis Factorial. Facultad de Ciencias Económicas y Empresariales UAM. Ciudad de México.

http://www.fuenterrebollo.com/economicas/econometri a/multivariante/factorial/analisis-factorial.pdf
Ríos Rodríguez, L. C., Carmona, E. A., y Ruvalcaba Arredondo, L. 
Escorsa, P., y Valls, J. (2003). Tecnología e Innovación en la empresa. Cataluña, España: Universitat Politècnica de Catalunya y Organización de Estados Iberoamericanos para la Educación, la Ciencia y la Cultura (OEI)

Fu, X., Mohnen, P., y Zanello, G. (2017). Innovation and productivity in formal and informal firms in Ghana. Elsevier, 131, 315-325.

https://doi.org/10.1016/j.techfore.2017.08.009

Fernández, C. (2013). Determinantes de la capacidad de innovación en pymes regionales. Revista de Administración de UFSM, 5, 749-765. https://doi.org/10.5902/198346597689

González, J., García, L., Caro C., y Romero, N. (2014). Estrategia y cultura de innovación, gestión de los recursos y generación de ideas: prácticas para gestionar la innovación en empresas. Revista pensamientos y gestión, 36(1), 107-133.

http://dx.doi.org/10.14482/pege.36.5567

González, O., y Mancillas, M. (2016). Propuesta de factores organizacionales en un Modelo de Innovación para PyMES: Empresas dedicadas a la elaboración de concreto en México. Memorias del XVIII concurso Lasallista de Investigación, Desarrollo e Innovación CLIDI

Haneda, S., e Ito, K. (2018). Organizational and human resource management and innovation: Which management practices are linked to product and/or process innovation? Research Polic, 47(1), 194-208. https://doi.org/10.1016/j.respol.2017.10.008

Helfat, C., Finkelstein, S., Mitchell, W., Peteraf, M., Singh, H., Teece, D., y Winter, S. (2007). Dynamic capabilities: Understanding strategic change in organizations. Malden, MA: Blackwell Publishing.

Ríos Rodríguez, L. C., Carmona, E. A., y Ruvalcaba Arredondo, L.
Hsu, Y. (2016). Innovation and competitive edge: effective design management in Chinese SMEs. International Journal of Organizational Innovation, 8(16), 68-78.

Instituto Nacional de Estadística Geografía e Informática (INEGI). (2018). Resultados de la encuesta nacional sobre productividad y competitividad de las micro, pequeñas y medianas empresas (ENAPROCE) 2018. México. https://www.inegi.org.mx/contenidos/saladeprensa/bol etines/2019/especiales/ENAPR OCE2018.pdf

Jiménez, A. (2016). Relaciones Universidad-Empresa: hacia una productividad basada en Innovación. Revista Gestión y Tendencias (GESTEN), 1(2), 7-10. https://doi.org/10.11565/gesten.v2i1.11

Kato-Vidal, E. (2019). Produtividade e inovação em pequenas e médias empresas. estud.gerenc. Revista Estudios Gerenciales, 35(150), 38-46. http://dx.doi.org/10.18046/j.estger.2019.150.2909.

Khan, Z., Hussain, M., Shahbaz, M., Yang, S., y Jiao, Z. (2020). Natural resource abundance, technological innovation, and human capital nexus with financial development: A case study of China. Resources Policy, 65, 1-13. https://doi.org/10.1016/j.resourpol.2020.101585

Lecert, M., y Omrani, N. (2019). SME Internationalization: the Impact of Information Technology and Innovation. Journal of the Knowledge Economy. https://doi.org/10.1007/s13132-018-0576-3

Li, D., Zhenga, M., Cao, C., Chen, X., Ren, S., y Huang, M. (2017). The impact of legitimacy pressure and corporate profitability on green innovation: Evidence from China top 100. Journal of Cleaner Production, 141, 41-49. https://doi.org/10.1016/j.jclepro.2016.08.123 
Lloret-Segura, S., Ferreres-Traver, A., HernándezBaeza, A., y Tomás-Marco, I. (2014). El análisis factorial exploratorio de los ítems: una guía práctica, revisada y actualizada. Anales de Psicología, 30(10), 1151-1169.

http://dx.doi.org/10.6018/analesps.30.3.199361

López, L. (2016). Principales prácticas de recursos humanos que apoyan la innovación en las PYMES: empresas de Jalisco y Cataluña (Tesis doctoral). Universidad Ramón Llull, Barcelona.

López, G., Maldonado, G., Pinzón S., y García R. (2015). Colaboración y actividades de innovación en Pymes. Revista de Contaduría y Administración, 61(3), 568-581. https://doi.org/10.1016/j.cya.2015.05.016

Maldonado, G., y Martínez, M. (2012). Gestión del conocimiento y crecimiento en la PYME manufacturera de Aguascalientes. Cuadernos de Administración, Universidad del Valle, 28(47), 25-36.

Martínez, J., y Romero, I. (2017). Determinants of innovativeness in SMEs: disentangling core innovation and technology adoption capabilities. Review of Managerial Science, 11(3), 543-569. https://doi.org/10.1007/s11846-016-0196-x

Medina, C., y Espinosa, M. (1994). La innovación en las organizaciones modernas. Universidad Autónoma de México. Ciudad de México. http://wwwazc.uam.mx/publicaciones/gestion/num5/doc06.htm

Mendoza, J., y Valenzuela, A. (2013). Aprendizaje, Innovación y Gestión Tecnológica en la pequeña empresa un estudio de las industrias metalmecánica y de tecnologías de información en Sonora. Contaduría y Administración, 59(4), 253-284. https://doi.org/10.1016/S0186-1042(14)70162-7
Monzón, A. (2015). Gestión de innovación en empresas de base tecnológica del sector hidráulico cubano. Revista Ingeniería Hidráulica y Ambiental, 36(1) 3-15.

Nieves, J., y Quintana, A. (2016). Human resource practices and innovation in the hotel industry: The mediating role of human capital. Sage Discipline Hubs, 18(1), 72-83. https://doi.org/10.1177/1467358415624137

OCDE, y Eurostat (2018). Manual de Oslo. Guía para la recogida e interpretación de datos sobre innovación. Cuarta Edición. París-Luxemburgo: Grupo Tragsa.

Palacios, P., y Saavedra, M. (2016). El desempeño exportador y la innovación como una estrategia de crecimiento para la PYME en México. Revista mexicana de economía y finanzas nueva época REMEF, 11(2), 21-38. https://doi.org/10.21919/remef.v11i2.84

Pearson R., y Mundform, D. (2010). Recommended Sample Size for Conducting.

Exploratory Factor Analysis on Dichotomous Data. Journal of Modern Applied Statistical Methods, 9(2), 359-368. https://doi.org/10.22237/jmasm/1288584240

Pérez, M., y Góngora, G. (2016). La innovación como factor clave de la competitividad en la PYME industrial. Un estudio empírico. Red Internacional de Investigadores en Competitividad, Memoria del IV Congreso.

Real Academia Española de la Lengua. (2019). Diccionario de la Lengua Española. Recuperado el 18 de agosto de 2020 de https://dle.rae.es/?id=LgzBfa6 
Revelle, W., y Zinbarg, R. (2009). Coefficients alpha, beta, omega, and the glb: comments on Sijtsma.

Psychometrika 74, 145-154.

https://doi.org/10.1007/s11336-008-9102-z

Ron, R., y Sacoto, V. (2017). Las PYMES ecuatorianas: su impacto en el empleo como contribución del PIB, PYMES al PIB total. Revista Espacios, 38(53), 15-24.

Solleiro, J., y Castañón, R. (2015). Competitividad y sistemas de innovación: los retos para la inserción de México en el contexto global. Globalización, ciencia y tecnología, 165-197.

Solleiro, J., y Terán, A. (2012). Buenas prácticas de gestión de la innovación en Centros de Investigación Tecnológica. México: Instituto de Investigaciones Eléctricas y Universidad Nacional Autónoma de México.
Soto, P., Popa, S., y Martínez, I. (2018). Information technology, knowledge management and environmental dynamism as drivers of innovation ambidexterity: a study in SMEs. Journal of Knowledge Management, 22(4), 824-849.

https://doi.org/10.1108/JKM-10-2017-0448

Tabachnick, B., y Fidell, L. (2007). Using Multivariate Statistics. USA: Pearson.

Taber, K. (2018). The Use of Cronbach's Alpha When Developing and Reporting Research Instruments in Science Education. Research in Science Education 48, 1273-1296. https://doi.org/10.1007/s11165-016-96022

Taborda, M., Nova, L., y Bohórquez, I. (2018). Importancia de las Pymes para el desarrollo económico de Chile en el siglo XXI. Punto de vista, 9(14), 1-18. https://doi.org/10.15765/pdv.v9i14.1179
Ríos Rodríguez, L. C., Carmona, E. A., y Ruvalcaba Arredondo, L.
Vol. 7, núm. 19 / enero - abril del 2022 DOI: https://doi.org/10.36791/tcg.v7i19.133 